International Journal of Pure and Applied Mathematics

Volume 83 No. 4 2013, 549-558

ISSN: 1311-8080 (printed version); ISSN: 1314-3395 (on-line version)

url: http://www.ijpam.eu

doi: http://dx.doi.org/10.12732/ijpam.v83i4.3

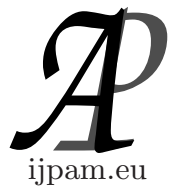

\title{
NUMERICAL SOLUTIONS OF SINGULAR PERTURBATION PROBLEMS WITH MULTIPLE BOUNDARY LAYERS AND INTERIOR LAYERS
}

\author{
Weiqun Zhang \\ Department of Mathematics \\ Wright State University \\ 3640, Colonel Glenn Hwy, Dayton, OH 45435, USA
}

\begin{abstract}
Two singular perturbation problems were considered: One with multiple boundary layers and one with interior layers. Numerical schemes were developed from the improved a'priori bounds. With a constant number of layer adapted grid points, numerical accuracy was maintained at the same level for a family of singular perturbation problems.
\end{abstract}

AMS Subject Classification: 65N12, 32E25

Key Words: singular perturbation, differential equations, boundary layers, interior layers, numerical solutions, stability

The study of singular perturbation problems is exceptionally useful because they describe the physics of many things of academic and economic interest. They may be used to model the weather, ocean currents, water flow in a pipe, the air's flow around a wing, and motion of stars inside a galaxy. The asymptotic expansion of O'Malley [8] and the a'priori bound theorem of Chang and Howes [1] were among the prominent approaches to solve singular perturbation problems. With the advance of unprecedented computing power, there has been a flow of literatures on numerical solutions from the nineteen eighties. Miller, O'Riordan and Shishkin [7] constructed the Shishkin-type mesh to

Received: October 4, 2012

(c) 2013 Academic Publications, Ltd. url: www.acadpubl.eu 
gain the independence of error estimation with respect to the singular perturbation parameter. Schultz and his students [2] and [4], successfully developed the stabilized high order finite difference methods. Lin, Schultz and Zhang [6] developed boundary layer detection theory from improved a'priori bounds for quasilinear singular perturbation problems.

Zhang, Schultz and Lin [12] developed sharp a'priori bounds for semi-linear singular perturbation problems including ones with multiple boundary layers. In this paper, we state one of the theorems in [12] and we study an example of a singular perturbation problem with such layers. Then we extend the boundary layer detection for quasi-linear singular perturbation problems in [6] to those with interior layers.

First, we consider singular perturbation problems in the following semilinear form,

$$
\begin{aligned}
& \varepsilon u "=g(x, u) \quad \text { for } \quad x \in(a, b), \\
& u(a)=v_{a} \quad \text { and } \quad u(b)=v_{b},
\end{aligned}
$$

where the singular perturbation parameter $\varepsilon \ll 1$ is a small positive constant and $g(x, u)$ is sufficiently continuously differentiable in the domain considered. In general, such a singular perturbation problem may display two boundary layers in its solution, and the width of each boundary layer is proportional to $\sqrt{\varepsilon}$.

In the following definition of stability of the solution $R(x)$ for the reduced equation $g(x, u)=0$ of the singular perturbation problem (1), assume that the function has the stated number of continuous partial derivatives with respect to $u$ in $\Omega(R)$. For an integer $q \geq 0$, the function $R=R(x)$ is said to be $\boldsymbol{I}_{\mathbf{q}}$-stable in $[a, b]$ if there exists a positive constant $m$ such that

$$
\partial_{u}^{j} g(x, R(x)) \equiv 0 \quad \text { for } \quad x \in[a, \quad b] \quad \text { and } \quad 0 \leq j \leq 2 q,
$$

and

$$
\partial_{u}^{2 q+1} g(x, u) \geq m \quad \text { in } \quad \Omega(R),
$$

Chang and Howes [1]. Here is one of the major theorems in [12]. It shows an improved a'priori bounds for the solution of the singular perturbation problem

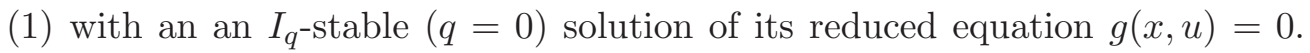
A summary of proof is provided to show that lengths of boundary layers are proportional to $\sqrt{\varepsilon}$. Details along with other $I_{q}$-stable cases can be found in $[12]$.

Theorem. Let $R(x)$ of class $C^{2}([a, b])$ be an an $I_{q}$-stable $(q=0)$ solution of the reduced equation $g(x, u)=0$ of the singular perturbation problem (1). 
Then there exist positive constants $C$ and $\varepsilon_{0}$ such that for $0<\varepsilon<\varepsilon_{0}$, it has a solution $u=u(x)$ which satisfies

$$
|u(x)-R(x)|<C \varepsilon \quad \text { for } \quad x \in[a+w \sqrt{\varepsilon}, b-w \sqrt{\varepsilon}],
$$

where $w$ is a positive constant for a family of small values of $\varepsilon$.

Proof. By Theorem 3.1 of Chang and Howes [1], there exists an $\varepsilon_{0}>0$ such that for $0<\varepsilon<\varepsilon_{0}$ the problem (1) has a solution $u=u(x)$ for $x \in[a, b]$ which satisfies for sufficiently small values of $\varepsilon \geq \varepsilon_{1}$.

$$
|u(x)-R(x)| \leq s(x)+t(x)+c \varepsilon,
$$

where $s(x)=\left|v_{a}-R(a)\right| e^{\frac{-\sqrt{m}(x-a)}{\sqrt{\varepsilon}}}$ and $t(x)=\left|v_{b}-R(b)\right| e^{\frac{-\sqrt{m}(b-x)}{\sqrt{\varepsilon}}}$.

We first consider the above positive function $s(x)=\left|v_{a}-R(a)\right| e^{\frac{-\sqrt{m}(x-a)}{\sqrt{\varepsilon}}}$. Choose a positive constant $w$, such that $w>-\frac{\ln \varepsilon_{1}}{\sqrt{m}}$. We can prove that $s(x)=$ $c_{1} e^{\frac{-\sqrt{m}(x-a)}{\sqrt{\varepsilon}}}<c_{1} \varepsilon$ for $x \in[a+w \sqrt{\varepsilon}, \quad b]$, where $c_{1}=\left|v_{a}-R(a)\right|$. For details, see $[12]$.

Similarly, we can prove that $t(x)=\left|v_{b}-R(b)\right| e^{\frac{-\sqrt{m}(b-x)}{\sqrt{\varepsilon}}}<c_{2} \varepsilon$ for $x \in$ $[a, b-w \sqrt{\varepsilon}]$., where $c_{2}=\left|v_{b}-R(b)\right|$. Thus, for $C=c_{1}+c_{2}+c$, we have

$$
|u(x)-R(x)|<C \varepsilon \quad \text { for } \quad x \in[a+w \sqrt{\varepsilon}, \quad b-w \sqrt{\varepsilon}] .
$$

Here is our first example with multiple boundary layers.

$$
\begin{aligned}
& \varepsilon u "=u \quad \text { for } \quad x \in(0,1), \\
& u(1)=1 \quad \text { and } \quad u(1)=2,
\end{aligned}
$$

O'Malley [8], Chang and Howes [1]. Two boundary layers, one at left and one at right are forced by boundary values. The exact solution $u$ is

$$
u(x)=\frac{\left(e^{\frac{-1}{\sqrt{\varepsilon}}}-2\right) e^{\frac{x}{\sqrt{\varepsilon}}}+\left(2-e^{\frac{1}{\sqrt{\varepsilon}}}\right) e^{\frac{-x}{\sqrt{\varepsilon}}}}{e^{\frac{-1}{\sqrt{\varepsilon}}}-e^{\frac{1}{\sqrt{\varepsilon}}}} .
$$

For small values of the singular perturbation parameter $\varepsilon$, it is approximately

$$
u(x) \approx 2 e^{\frac{-(1-x)}{\sqrt{\varepsilon}}}+e^{\frac{-x}{\sqrt{\varepsilon}}},
$$

since $e^{\frac{-1}{\sqrt{\varepsilon}}} \approx 0$ and $\frac{2-e^{\frac{1}{\sqrt{\varepsilon}}}}{e^{\frac{-1}{\sqrt{\varepsilon}}}-e^{\frac{1}{\sqrt{\varepsilon}}}} \approx 1$. 


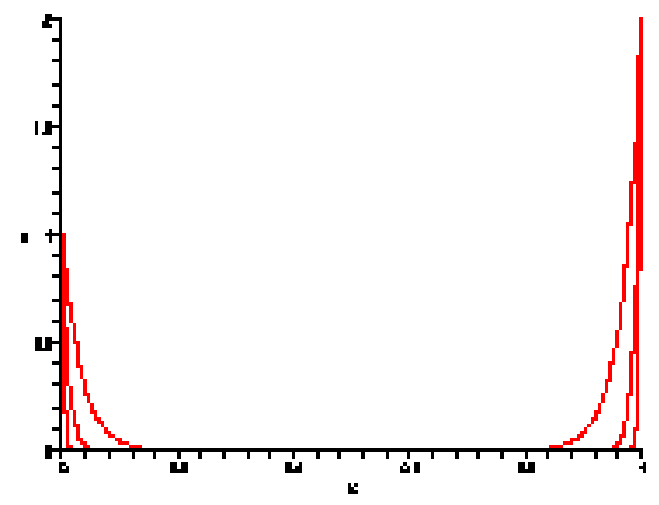

Figure 1.1: Graphs of the solutions of the problem $(2), \varepsilon=.001, .0001$ and .0001

The approximation form of $u$ confirms the observation of multiple boundary layers at both boundaries $x=0$ and $x=1$. Let us examine the solution more closely. The solution $u$ is defined for all $\varepsilon>0$ and moreover, $\lim _{\varepsilon \rightarrow 0^{+}} u(x, \varepsilon)=0$ for $\delta \leq \mathrm{x} \leq 1-\delta$, where $\delta$ is a fixed constant $(0,1)$. The function $u(x)$ attains its limiting value non-uniformly in the neighborhoods of $x=0$ and $x=1$ in the sense that

$$
\lim _{\varepsilon \rightarrow 0^{+}} \lim _{x \rightarrow 0^{+}} u(x, \varepsilon)=1 \neq 0=\lim _{x \rightarrow 0^{+}} \lim _{\varepsilon \rightarrow 0^{+}} u(x, \varepsilon)
$$

and

$$
\lim _{\varepsilon \rightarrow 0^{+}} \lim _{x \rightarrow 1^{-}} u(x, \varepsilon)=2 \neq 0=\lim _{x \rightarrow 1^{-}} \lim _{\varepsilon \rightarrow 0^{+}} u(x, \varepsilon) .
$$

The graphs of $u$ for small values of $\varepsilon$ are shown in Figure 1.1.

Note that by setting $\varepsilon=0$ we obtain the reduced equation $u \equiv 0$ which is the approximation of the singular perturbation problem on its interior domain.

For the length of left boundary layer, we have

$$
e^{\frac{-x}{\sqrt{\varepsilon}}}<\varepsilon \quad \Leftrightarrow \quad \frac{-x}{\sqrt{\varepsilon}}<\ln \varepsilon \quad \Leftrightarrow \quad x>(-\ln \varepsilon) \sqrt{\varepsilon}
$$

$w=30 \geq-\ln \varepsilon \quad$ for $\varepsilon>10^{-12}$ as shown in the proof of the theorem. Similarly, for the length of the right boundary layer, we have

$$
e^{-\frac{1-x}{\sqrt{\varepsilon}}}<\varepsilon \quad \Leftrightarrow \quad-\frac{1-x}{\sqrt{\varepsilon}}<\ln \varepsilon \quad \Leftrightarrow \quad x<1-(-\ln \varepsilon) \sqrt{\varepsilon},
$$




\begin{tabular}{|l|l|l|l|}
\hline Number of points & 200 & 400 & 1000 \\
\hline Maximal error & $3.44^{*} 10^{-4}$ & $8.61^{*} 10^{-5}$ & $1.38^{*} 10^{-5}$ \\
\hline
\end{tabular}

Table 1.1: The convergence of the new method on the left boundary layer, $\varepsilon=10^{-6}$

$w=30 \geq-\ln \varepsilon$ for $\varepsilon>10^{-12}$. Therefore, the exact solution $u<C \varepsilon$ for $\varepsilon>10^{-12} w \sqrt{\varepsilon} \leq x \leq 1-w \sqrt{\varepsilon}$ and a positive constant $C$. It follows that the lengths of the boundary layers are proportional to $\sqrt{\varepsilon}$. The proportion constant $w=30$ is the boundary layer parameter. The singular perturbation problem (1) is approximated by following three problems,

$$
\begin{gathered}
\varepsilon u "=u \text { for } \quad x \in\left(0, \quad t_{1}\right), \\
u(1)=1 \text { and } u\left(t_{1}\right)=0, \\
u=0 \text { for } \leq x \in\left(t_{1}, \quad t_{2}\right),
\end{gathered}
$$

and

$$
\begin{gathered}
\varepsilon u "=u \quad \text { for } \quad x \in\left(\begin{array}{l}
0, \quad t_{1}
\end{array}\right), \\
u\left(t_{2}\right)=0 \quad \text { and } \quad u(1)=2, \\
\text { where } t_{1}=w \sqrt{\varepsilon} \text { and } t_{2}=1-w \sqrt{\varepsilon},
\end{gathered}
$$

We apply the second order central differences on the following boundary layer adapted mesh to solve the singular perturbation problems (1.1) and (1.3), while for (1.2), $u$ is given explicitly or implicitly from the reduced equation $g(x, u)=$ 0 . Figure 1.2 displays the layer adapted grid points at both boundaries.

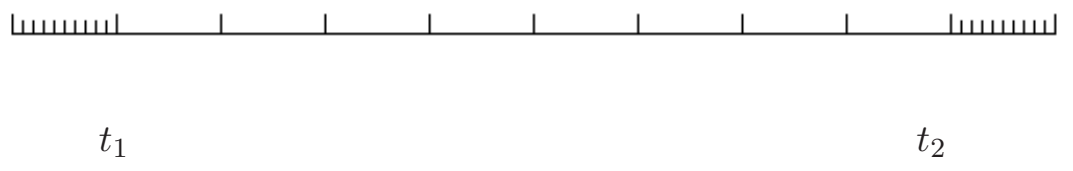

Figure 1.2: The boundary layer adapted mesh at both boundaries

Table 1.1 shows the convergence of the new method with central finite differences on the left boundary layer as the number of mesh points increases. Table 1.2 shows the convergence of the new method on the right boundary layer as the number of mesh points increases. 


\begin{tabular}{|l|l|l|l|}
\hline Number of points & 200 & 400 & 1000 \\
\hline Maximal error & $6.88^{*} 10^{-4}$ & $1.72^{*} 10^{-4}$ & $2.76^{*} 10^{-5}$ \\
\hline
\end{tabular}

Table 1.2: The convergence of the new method on the right boundary layer, $\varepsilon=10^{-\mathbf{6}}$

\begin{tabular}{|l|l|l|l|}
\hline \multirow{2}{*}{ Number of mesh points } & \multicolumn{3}{|l|}{ Maximal Error } \\
\cline { 2 - 4 } & $\varepsilon=10^{-6}$ & $\varepsilon=10^{-8}$ & $\varepsilon=10^{-10}$ \\
\hline $\begin{array}{l}N_{b}=500 \text { for the left boundary } \\
\text { layer }\end{array}$ & $5.52^{*} 10^{-5}$ & $5.52^{*} 10^{-5}$ & $5.52^{*} 10^{-5}$ \\
L.B.L. & & & \\
\hline $\begin{array}{l}N_{b}=500 \text { for the right boundary } \\
\text { layer }\end{array}$ & $1.10^{*} 10^{-4}$ & $1.10^{*} 10^{-4}$ & $1.10^{*} 10^{-4}$ \\
\hline $\begin{array}{l}N_{b}=1000 \text { for the left boundary } \\
\text { layer } \\
\text { L.B.L. }\end{array}$ & $1.38^{*} 10^{-5}$ & $1.38^{*} 10^{-5}$ & $1.38^{*} 10^{-5}$ \\
\hline $\begin{array}{l}N_{b}=1000 \text { for the right boundary } \\
\text { layer }\end{array}$ & $2.76^{*} 10^{-5}$ & $2.76^{*} 10^{-5}$ & $2.76^{*} 10^{-5}$ \\
\hline
\end{tabular}

Table 1.3: Table 1.3. The robustness of the new method

Table 1.3 shows the robustness of the separation method. For a family of diminishing values of the singular perturbation parameter, the error is stabilized for a fixed number of mesh points on both boundary layers.

Next, we extend the layer detection for quasi-linear singular perturbation problems in [6] to those with interior layers. We consider singular perturbation problems in the quasi-linear form,

$$
\begin{aligned}
& \varepsilon u "=f(x, u) u^{\prime}+g(x, u) \text { for } \quad x \in(a, b) \text { and } f(x, u) \neq 0, \\
& u(a)=v_{a} \text { and } u(b)=v_{b}
\end{aligned}
$$

where $f$ and $g$ are continuous. By the improved a'priori bounds of Zhang [11], if $f(x, u) \leq-k<0$ for a positive constant $k$ and $x \in(a, b)$, it can be analytically approximated by the two differential equations.

$$
\begin{aligned}
& f(x, u) u^{\prime}+g(x, u)=0 \text { for } \quad x \in(t, b), \\
& u(b)=v_{b},
\end{aligned}
$$


and

$$
\begin{aligned}
& \varepsilon u^{\prime \prime}=f(x, u) u^{\prime}+g(x, u) \text { for } x \in(a, t), \\
& u(a)=v_{a} \text { and } u(t)=v_{t},
\end{aligned}
$$

where the turning point is $t=a+w \varepsilon$ and $w$ is a constant for a family of values of $\varepsilon$.

If $f(x, u) \geq k>0$ for a positive constant $k$ and $x \in(a, b)$, the singular perturbation problem (2) can be analytically approximated by the following two differential equations.

$$
\begin{aligned}
& f(x, u) u^{\prime}+g(x, u)=0 \quad \text { for } \quad x \in(a, t), \\
& u(a)=v_{a},
\end{aligned}
$$

and

$$
\begin{aligned}
& \varepsilon u^{\prime \prime}=f(x, u) u^{\prime}+g(x, u) \text { for } \quad x \in(t, b), \\
& u(t)=v_{t} \text { and } u(b)=v_{b},
\end{aligned}
$$

where the turning point is $t=b-w \varepsilon$ and $w$ is a constant for a family of values of $\varepsilon$. Note that the boundary value $v_{t}$ at the transition point $t$ of the equations (2.2) and (2.4) is not known. Let $R_{b}(x)$ be the solution of $(2.1)$ and let $R_{a}(x)$ be the solution of (2.3). We substitute $R_{b}(t)$ for $v_{t}$ into equation (2.2) and substitute $R_{a}(t)$ for $v_{t}$ into equation (2.4) respectively.

Here is an example of quasi-linear singular perturbation problems with interior layers.

$$
\begin{array}{ll}
\varepsilon u "=u u^{\prime}-u^{3} & \text { for } \quad x \in(0,1), \\
u(1)=\frac{2}{3} \quad \text { and } & u(1)=-\frac{1}{2},
\end{array}
$$

from Smith [9] and Whitman [10]. It is known that a shock occurs as the singular parameter $\varepsilon$ decreases. As a result, the interior layer is displayed in its solution.

As the singular perturbation vanishes, for $u \neq 0$, we have $u^{\prime}=u^{2}$ whose general solution is $u(x)=\frac{1}{c-x}$ where $c$ is constant. At the left boundary $x=0$, $c$ is $\frac{3}{2}$ since $u(1)=\frac{2}{3}$. At the right boundary $x=1, c$ is -1 since $u(1)=-\frac{1}{2}$. Let $R_{a}$ and $R_{b}$ be the solutions of the reduced differential equation to the left boundary and the right boundary respectively. Then we have,

$$
R_{a}(x)=\frac{1}{\frac{3}{2}-x}=\frac{2}{3-2 x}, \text { and } R_{b}(x)=\frac{1}{-1-x}=-\frac{1}{1+x} .
$$

Further analysis shows that the interior boundary layer or the shock occurs at the unique point $x_{s}$ such that $R_{a}\left(x_{s}\right)+R_{b}\left(x_{s}\right)=0$. Thus, we have

$$
R_{a}\left(x_{s}\right)+R_{b}\left(x_{s}\right)=0 \quad \Leftrightarrow \quad \frac{2}{3-2 x_{s}}+\left(-\frac{1}{1+x_{s}}\right)=0 \quad \Leftrightarrow \quad x_{s}=\frac{1}{4} .
$$




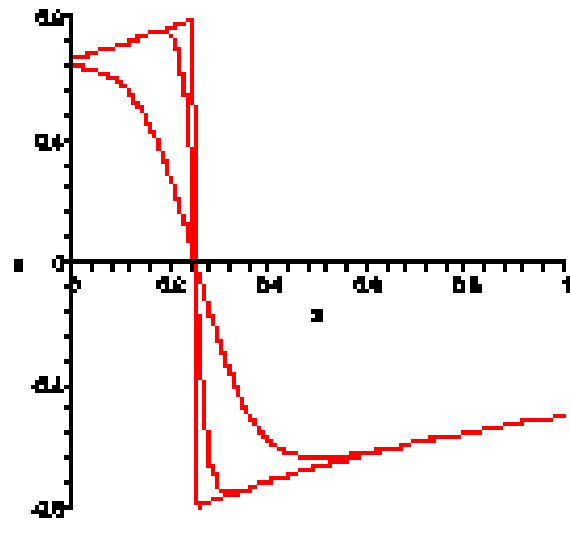

(a)

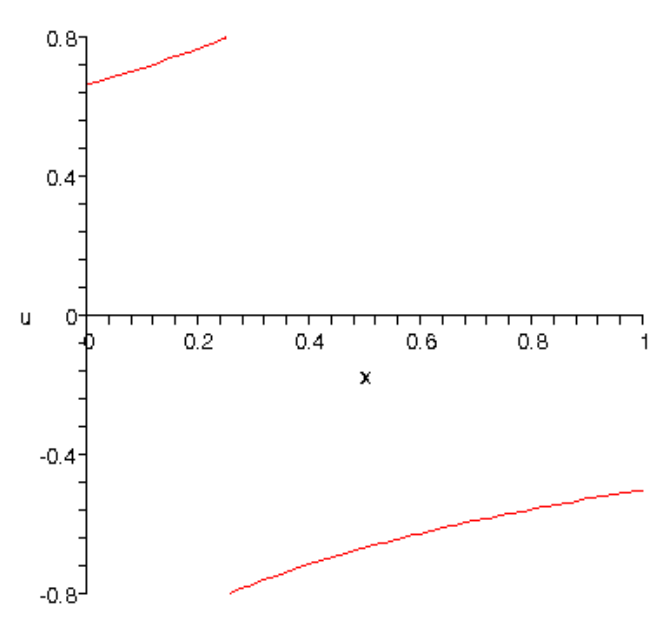

(b)

Figure 2.3: Graphs of solutions of the problem (3) and its reduced equations; (a) from left to right $\varepsilon=.05, .01$ and .001; (b)the reduced equations

Now, the singularly perturbed differential equation (3) is equivalent to

$$
\begin{array}{ll}
\varepsilon u "=u u^{\prime}-u^{3} & \text { for } \quad x \in\left(0, x_{s}\right), \\
u(1)=\frac{2}{3} \quad \text { and } & u\left(x_{s}\right)=0
\end{array}
$$

and

$$
\begin{aligned}
& \varepsilon u "=u u^{\prime}-u^{3} \quad \text { for } x \in\left(x_{s}, 1\right), \\
& u\left(x_{s}\right)=0 \text { and } u(1)=-\frac{1}{2} .
\end{aligned}
$$

The equation (3.1) has a boundary layer at the right boundary $x_{s}=\frac{1}{4}$ while the equation (3.2) has one at left $x_{s}=\frac{1}{4}$. The interior boundary layer of the singular perturbation problem (3) is formed by the coexistence of the above two boundary layers, Figure 2.1.

In general, a reduced equation can be solved numerically with high order Runge Kutta methods. For (3.1) and (3.2), their reduced equations are solved analytically above. On their boundary layer domains, each is solved with the second order central finite difference approximation. The stability of a boundary value perturbation for such problems is proved in Zhang [11]. On the non layer domain, we get almost perfect results from the reduced equations. On the layer 
domain, the maximal error is controlled at $10^{-4}$ level for $\varepsilon=10^{-5}$ by using a few hundred mesh points, Table 2.1.

\begin{tabular}{|l|l|l|l|}
\hline Number of points on the layer & 200 & 400 & 600 \\
\hline Maximal error & $1.35^{*} 10^{-3}$ & $2.91^{*} 10^{-4}$ & $2.51^{*} 10^{-4}$ \\
\hline
\end{tabular}

Table 2.4: The convergence of the new method, $\varepsilon=10^{-\mathbf{5}}$

For a family of extremely small $\varepsilon \leq 10^{-10}$ values, we controlled the error at $10^{-3}$ level with 200 mesh points and at $10^{-4}$ level with 400 points, Table 2.2 .

\begin{tabular}{|l|l|l|l|l|l|l|}
\hline $\begin{array}{l}\text { Number } \\
\text { of Points } \\
\text { on the } \\
\text { boundary } \\
\text { layer }\end{array}$ & \multicolumn{5}{|c|}{ Maximal Error } \\
\cline { 2 - 6 } & $\varepsilon=10^{-5}$ & $\varepsilon=10^{-6}$ & $\varepsilon=10^{-7}$ & $\varepsilon=10^{-8}$ & $\varepsilon=10^{-9}$ & $\varepsilon=10^{-10}$ \\
\hline 200 & $1.35^{*} 10^{-3}$ & $1.69^{*} 10^{-3}$ & $1.72^{*} 10^{-3}$ & $1.72^{*} 10^{-3}$ & $1.72^{*} 10^{-3}$ & $1.72^{*} 10^{-3}$ \\
\hline 400 & $2.91^{*} 10^{-4}$ & $3.77^{*} 10^{-4}$ & $4.07^{*} 10^{-4}$ & $4.10^{*} 10^{-4}$ & $4.10^{*} 10^{-4}$ & $4.10^{*} 10^{-4}$ \\
\hline
\end{tabular}

Table 2.5: The maximal error of the new method

\section{References}

[1] K.W. Chang, F.A. Howes, Nonlinear Singular Perturbation Phenomena: Theory and Application, Spring-Verlag (1984).

[2] J.Y. Choo, D.H. Schultz, High order methods for differential equations with small coefficients for the second order terms, Computers Math. Applic., 25, No. 1 (1993), 105-123.

[3] M.H. Holmes, Introduction to Perturbation Methods, Springer-Verlag (1995).

[4] F.O. Ilicasu, D.H. Schultz, High order methods for singular perturbation problems, Computers Math. Applic., 47 (2002), 391-417.

[5] H.B. Keller, Numerical Methods for Two-Point Boundary Value Problems, Blaisdell Publishing Company (1968). 
[6] T.C. Lin, D.H. Schultz, W. Zhang, Numerical solutions of linear and nonlinear singular perturbation problems, Computers Math. Applic., 55, No. 11 (2008), 2574-2592.

[7] J.J.H. Miller, E. O'Riordan, G.I. Shishkin, Fitted Numerical Methods for Singular Perturbation Problems, World Scientific (1996).

[8] R.E. O’Malley Jr., Introduction to Singular Perturbations, Academic Press, New York (1974).

[9] D.R. Smith, Singular Perturbation Theory an Introduction with Applications, Cambridge University Press (1985).

[10] G.B. Whitman, Linear and Nonlinear Waves, Wiley, New York (1974).

[11] W. Zhang, Numerical Solutions of Linear and Nonlinear Singular Perturbation Problems, Ph.D. Thesis, University of Wisconsin Milwaukee (2006).

[12] W. Zhang, David H. Schultz, T.C. Lin, Sharp bounds for semilinear singular perturbation problems, International Journal of Applied Sciences and Computing, 18, No. 1 (2012). 